\title{
Aspectos reproductivos de Chicoreus brevifrons (Lamarck, 1822) (Neogastropoda: Muricidae) de la laguna de La Restinga, isla de Margarita, Venezuela
}

\author{
Reproductive aspects of Chicoreus brevifrons (Lamarck, \\ 1822) (Neogastropoda: Muricidae) from La Restinga lagoon, \\ Margarita Island, Venezuela
}

\author{
Ana G. Maldonado ${ }^{1 *}$, Roberta Crescini $^{1}$, William Villalba ${ }^{1}$ y Yuruani Fuentes ${ }^{1}$
}

\begin{abstract}
RESUMEN
Chicoreus brevifrons se caracteriza por ser carnívoro y necrófago, relativamente abundante en las costas venezolanas donde reviste importancia económica y ecológica por ser una especie depredadora de ostras y otros moluscos en cultivos y ambientes marinos. El presente trabajo tuvo la finalidad de analizar algunos aspectos reproductivos de la especie en la laguna de La Restinga, isla de Margarita, Venezuela, en cuatro estaciones de esta, desde la zona más interna a la zona más externa. Se recolectaron muestras mensualmente para determinar la proporción por sexos; además, fueron extraídas del medio algunas posturas para su descripción y la observación del crecimiento inicial de la especie. C. brevifrons se caracteriza por ser un organismo dioico, claramente diferenciable por la presencia de pene en los machos y de vagina y cápsula de la albúmina en las hembras. La proporción sexual general no se alejó de la relación 1:1, a pesar de que algunos meses las hembras predominaron sobre los machos. La postura de $C$. brevifrons es de tipo racimo, con 65 a 165 pequeñas cápsulas de color blanco a la puesta. Mientras los organismos se desarrollan intracapsularmente, estos se observan de color marrón al momento de la eclosión, la cual se efectúa a partir de los 45-50 días desde la puesta. Las tallas iniciales estuvieron entre $1.60 \mathrm{~mm}$ y $2.56 \mathrm{~mm}$ de LT. Se recomienda continuar los estudios poblacionales y biológicos de esta especie que permitan conocer más acerca de su etología, importancia comercial y cuánto influyen sobre los cultivos de otros moluscos.
\end{abstract}

Palabras claves: Mollusca, gasterópodos, proporción sexual, eclosión, crecimiento.

\begin{abstract}
Chicoreus brevifrons is characterized by being carnivorous, necrophagous, and relatively abundant in Venezuelan coasts, where it has an economic and ecological importance since it preys on oysters and other mollusks in marine environments and culture systems. This study aims at analyzing some reproductive aspects of the species in La Restinga lagoon, Margarita Island, Venezuela, at four stations from inside to outside of this lagoon. Samples were taken monthly and were used to determine sex ratio; in addition, some eggs were also taken from the site, for description and observation of initial growth of the species. C. brevifrons is characterized for being dioecious, clearly distinguishable for the presence of a penis in males and a vagina and albumin capsule in
\end{abstract}

Escuela de Ciencias Aplicadas del Mar, Universidad de Oriente, isla de Margarita, Venezuela.

anagabrielamaldonado@gmail.com*; robertacrescini@gmail.com; wvillalbaluna@gmail.com; yuru.fuentes@gmail.com

Recibido: 11 de marzo de 2015

Corregido: 29 de junio de 2015

Aceptado: 22 de noviembre de 2015

DOI: http://dx.doi.org/10.15359/revmar.8-1.3

Rev. Mar. Cost. ISSN 1659-455X. Vol. 8 (1): 41-50, Enero-Junio 2016. 
females. In general, sex ratio was 1:1, although females were more abundant than males during some months. Eggs from C. brevifrons are cluster-like, with 65 to 165 small white capsules. While the organisms develop inside the capsules, they are brown upon spawning, which takes place 45-50 days after they are laid. Initial sizes were between $1.60 \mathrm{~mm}$ and $2.56 \mathrm{~mm}$ of TL. Further biological and populations studies of this species are recommended in order to know more about its ethology, commercial importance and how they affect mollusk cultures.

Keywords: Mollusca, gastropods, sexual proportion, spawning, growth.

\section{INTRODUCCIÓN}

En las costas orientales de Venezuela Chicoreus brevifrons es conocido vulgarmente como chirigua, chivato o arrechón (Itriago, 1977) y se caracteriza por poseer una concha grande (de aproximadamente 200 $\mathrm{mm}$ de Lt), ahusada, de espiral alta; columnela lisa; labio externo sinuoso y dentellado; canal sifonal oblicuo, bordeado por espinas, algo curvado hacia el dorso; várices robustas, con espinas foliadas y gruesas (Marval, 1981; Nieto-Bernal et al. 2011). Puede presentar una gran variación en su coloración y en el desarrollo de los procesos espinosos, en función de las diferentes condiciones del medio en el que habita (Díaz \& Puyana, 1994).

La alta disponibilidad trófica en las áreas de manglar Rhizophora mangle (mangle rojo) constituye un buen sustrato para los organismos sésiles (Prüsmann \& Palacio, 2008), brinda protección a las larvas y juveniles de numerosas especies de invertebrados como el C. brevifrons (Quinceno \& Palacio, 2008), lo que hace de estas áreas de aguas someras su hábitat (Marval, 1981; D'Armas et al. 2009). Estos hábitats naturales facilitan el crecimiento y desarrollo de la especie, permiten su amplia distribución y disponen del alimento necesario.

Los factores que determinan el comportamiento reproductivo para especies de moluscos son de variada naturaleza, por ello es importante la realización de muestreos que permitan la recolección de información para desarrollar estudios biológicos (Tresierra \& Culquichicón, 1993; Baqueiro \& Aldana, 2003).

Muchas especies de la familia Muricidae son de importancia comercial, siendo explotadas por los pescadores con fines de consumo y para uso artesanal de la concha. El estudio de la eclosión, cápsulas de huevos y embriones de gasterópodos es importante no solo para conocer la biología reproductiva en general, sino también por sus implicaciones para la dispersión, la biogeografía y la taxonomía (Mostafa et al. 2013). En Venezuela se han desarrollado diversas investigaciones sobre $C$. brevifrons, entre las que se pueden señalar el trabajo de Itriago (1977) quien efectuó un estudio citogenético y anatómico de la especie; Marval (1981) realizó estudios comparativos del tracto digestivo y contribuyó a la biología de $C$. brevifrons y Chicoreus pomun; Cabrera et al. 
(1990) evalúan su biología; Miloslavich et al. (2007), analizando el imposex en gasterópodos, consideran la especie como propensa a este fenómeno; Galindo (2009) hizo estudios de dimorfismo; D'Armas et al. (2009) determinaron la composición de ácidos grasos y Ordaz et al. (2010) investigaron los metabolitos secundarios de la especie. Dada la importancia económica y ecológica que tiene esta especie por ser depredadora de ostras y otros moluscos en los cultivos $\mathrm{y}$ ambientes marinos, se evaluaron aspectos reproductivos de la especie, con la finalidad de aportar información actualizada que sirva de base para sugerir estrategias de manejo sustentable, así como considerar el potencial de cultivo de C. brevifrons en Venezuela.

\section{MATERIALES Y MÉTODOS}

\section{Área de estudio y muestreo:}

El Parque Nacional Laguna de La Restinga se encuentra ubicado entre los $10^{\circ} 58^{\prime}$ y $11^{\circ} 05^{\prime}$ de latitud norte y los $64^{\circ} 01^{\prime}$ y $64^{\circ} 17^{\prime}$ de longitud oeste, en el istmo que une la península de Macanao con el sector oriental de la isla de Margarita, Estado Nueva Esparta. La laguna está separada del mar Caribe por una barra arenosa o restinga de 23 kilómetros de largo y unos 50 metros de ancho, y es la única unión natural entre la isla de Margarita y la península de Macanao (Ramírez, 1996). Se realizaron muestreos mensuales en cuatro estaciones de la laguna de La Restinga: El Pato, Mánamo, El Gato y El Indio, considerando el orden de estas estaciones desde la zona más interna de la laguna hacia la zona más externa de esta (Fig. 1). En cada salida se recolectaron, por medio de buceo libre, ejemplares de C. brevifrons de manera aleatoria, para poder obtener animales de todas las tallas observadas en el medio. Estos fueron extraídos del sustrato duro (raíces de manglar) y del sustrato fangoso, guardados en bolsas previamente identificadas con la estación respectiva, para su posterior traslado al laboratorio.

Proporción sexual: La proporción sexual general se obtuvo a partir de muestras mensuales de la especie. Las conchas de los caracoles Murícidos carecen de dimorfismo sexual (Naegel \& Gómez del Prado, 2004), por lo que para la determinación del sexo es necesario que los individuos vengan extraídos de las conchas para observar el sexo de cada uno, identificando macroscópicamente la presencia del pene en los machos y la ausencia de este carácter en las hembras, quienes presentan la cápsula de la albúmina, la glándula de la cápsula y la vagina (Miloslavich et al. 2007). La proporción sexual, por meses y total, se analizó mediante la prueba de Ji-cuadrado (Zar, 1996) para comprobar si esta se aleja significativamente de la relación 1:1.

Evaluación del desarrollo de la postura y crecimiento inicial: Fueron extraídas del medio algunas posturas para su descripción y la observación del crecimiento inicial de los juveniles. Las posturas se fotografiaron en el medio y se colocaron en recipientes con agua para conservarlas durante el traslado al laboratorio, procurando 


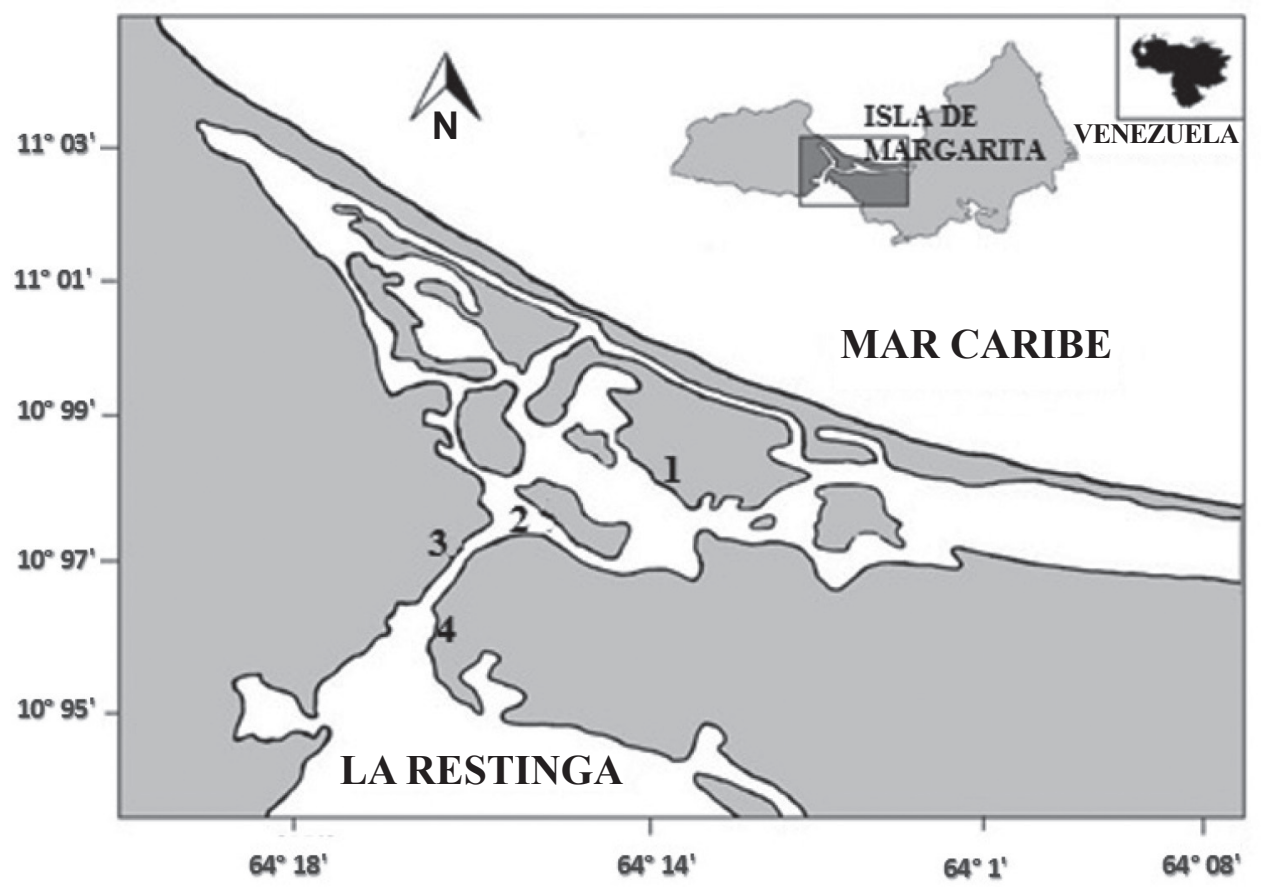

Fig. 1. Ubicación geográfica relativa de las estaciones de muestreo en: 1) El Pato, 2) Mánamo, 3) El Gato y 4) El Indio en la laguna de La Restinga, Venezuela

Fig. 1. Relative location of sampling stations: 1) El Pato, 2) Mánamo, 3) El Gato, and 4) El Indio at La Restinga lagoon, Venezuela

mantener constante la temperatura del agua. Asimismo, las cápsulas se extrajeron del acuario a medida que se observaban cambios de coloración en estas, manteniendo este procedimiento hasta el momento de la eclosión, además de registrar el tiempo de la eclosión y el crecimiento inicial con mediciones en la longitud total cada 5 días, tiempo establecido luego de un estudio previo.

\section{RESULTADOS Y DISCUSIÓN}

Proporción sexual: Se recolectó un total de 381 ejemplares de $C$. brevifrons con tallas comprendidas entre los 40 y $140 \mathrm{~mm}$, respectivamente
$(69.37 \pm 28.00)$, de los cuales 224 fueron hembras $(58.79 \%)$ y 157 machos $(41.21 \%)$, siendo las hembras más frecuentes que los machos. De manera general, la proporción sexual no se desvió de la razón 1:1, a pesar de que en los meses de abril y julio las hembras predominaron sobre los machos (Fig. 2; Cuadro 1).

La proporción entre sexos estimada de manera general para $C$. brevifrons no se alejó significativamente de la razón 1:1, concordando con Marval (1981), quien obtuvo una proporción 1:1 para la misma especie en Mochima, Península de Araya, atribuyendo este resultado a que la especie se 


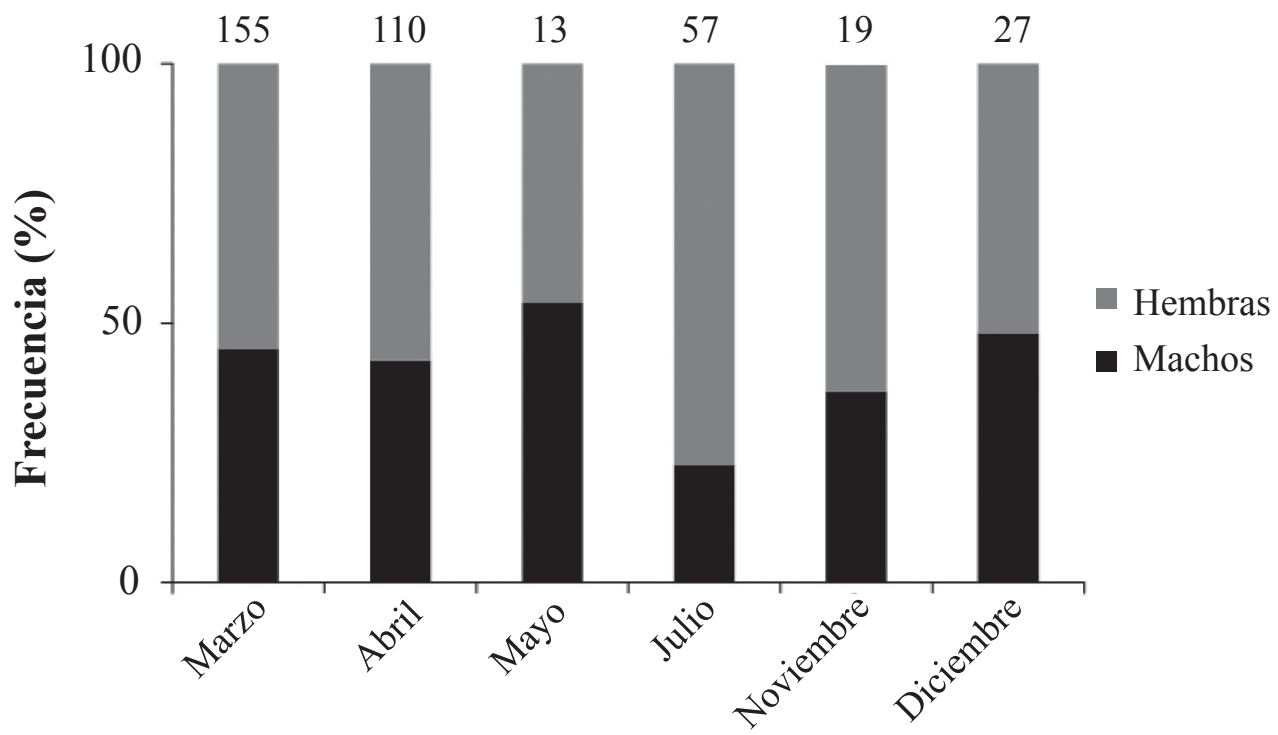

Hig. '2. Proporcion sexual de la especie C'hicoreus brevifrons de la laguna de La Kestinga, isla de Margarita, Venezuela

Fig. 2. Sex ratio of the species Chicoreus brevifrons from La Restinga lagoon, Margarita Island, Venezuela

Cuadro 1. Análisis Ji-cuadrado y proporción sexual en los meses de estudio de Chicoreus brevifrons Table 1. Chi-square test and sex ratio in the months of study of Chicoreus brevifrons

\begin{tabular}{cccccc}
\hline Meses & Fo (machos) & Fo (hembras) & N & X2 & $\boldsymbol{P}$ \\
\hline marzo & 70 & 85 & 155 & 1.45 & $>0.05$ \\
abril & 47 & 63 & 110 & 2.32 & $<0.05$ \\
mayo & 7 & 6 & 13 & 0.07 & $>0.05$ \\
julio & 13 & 44 & 57 & 16.85 & $<0.05$ \\
noviembre & 7 & 12 & 19 & 1.31 & $>0.05$ \\
diciembre & 13 & 14 & 27 & 0.03 & $>0.05$ \\
Totales & 157 & 224 & 381 & 11.78 & $>0.05$ \\
\hline
\end{tabular}

encontraba en época reproductiva y las proporciones de sexos se igualan. Es probable que nuestros resultados se deban a que los meses en los que las hembras son más abundantes (marzo, abril, julio y noviembre), con respecto a los machos, representan las épocas de desove. Por otra parte, se han publicado resultados diferentes en otras especies de Murícidos, Olabarria (1999) obtuvo una proporción de sexos significativamente diferente de 1:1 para la especie Hexaplex erythrostomus, así como Morfín (2000) la obtuvo para Plicopurpura pansa, concluyendo que la proporción sexual puede estar 
afectada por cambios en la distribución espacial de los organismos.

Desarrollo de Chicoreus brevifrons: En la figura 3 se observa la postura realizada por Chicoreus brevifrons. Esta consiste en un racimo, con 65 a 165 pequeñas cápsulas (de colorblanco recién puestas) (Fig. 3-A), donde cada cápsula tiene una apariencia fusiforme, con la porción superior sellada por un tapón mucoso que se rompe al momento de la eclosión y dentro de ellas se encuentran los huevos fecundados. A medida que transcurren los días y los individuos se van desarrollando intracapsularmente, las cápsulas externas van oscureciendo su coloración a un tono más amarillento $\mathrm{y}$ en las internas se observan pequeñas masas de color lila (Fig. 3-B y C).

Una característica resaltante de la especie estudiada es su capacidad para reproducirse a través de la fecundación interna y la realización de la ovoposición de sus huevos en cápsulas especiales. Según Román et al. (2001), el número de huevos por cápsula varía entre las especies y esto puede deberse al tamaño de los adultos o a las condiciones del medio ambiente. El tipo de postura coincide con las descripciones de Mostafa et al. (2013) para la especie Chicoreus ramosus, la cantidad de individuos que se originan de cada cápsula es igual a la reportada por González (1970), aunque, por otra parte, Cabrera et al. (1990) señalan la presencia de dos a cuatro huevos por cápsula, siendo el número total de cápsulas de 40 para la misma especie en Venezuela. Mostafa et al. (2013), en $C$. ramosus en condiciones de laboratorio, reportaron un total de 12 a 27 huevos desarrollados en forma de embrión y el resto pasó a ser material nutricio.

A los 21 días de desarrollo comienza a notarse la presencia de los huevos nutricios, que son aquellos huevos que no alcanzan eficazmente su desarrollo dentro de la cápsula, teniendo la misma apariencia inicial; por otra parte, los huevos que obtienen un buen crecimiento son más grandes, con una forma más asimétrica por la iniciación del proceso de torsión y se puede notar la neoformación de la concha (Fig. 3-D y E).

Roman et al. (2001) y Cognetti et al. (2008) mencionan que los huevos no viables son una fuente de alimento importante para los organismos que están creciendo y desarrollándose dentro de la masa ovígera. A su vez, Leiva et al. (1998) consideran que el tiempo para que los embriones comiencen a ingerir los huevos nutricios es a partir de $\operatorname{los} 21$ a 25 días de desarrollo.

A partir del día 42, los individuos se dispersan hacia el interior de la masa ovígera y presentan una concha bien desarrollada, dentro de las cápsulas se observan de color marrón, siendo esta su apariencia final antes de la eclosión, la cual se efectúa a partir de los 4550 días desde la puesta, donde cada cápsula da origen de 3 a 5 individuos. Las tallas iniciales estuvieron comprendidas entre 1.60 y $3.90 \mathrm{~mm}$ de longitud total (Lt), presentando a los 15 días posteriores a la eclosión, tallas promedio de $4.60 \mathrm{~mm}$ y a los 30 días, longitudes totales promedio de 5.48 mm; además de observarse la concha 


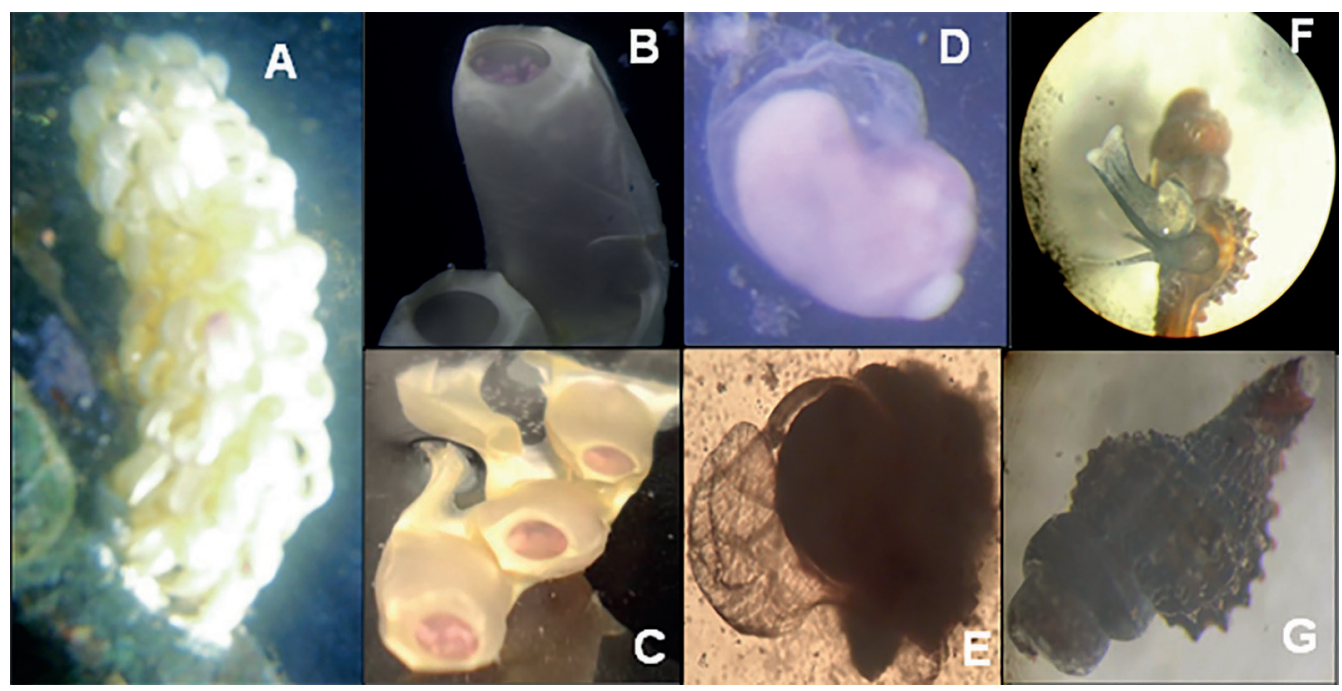

Fig. 3. A.- Masa ovígera de Chicoreus brevifrons. B. y C.- Cápsulas de la masa ovígera. D.- Huevo de Chicoreus brevifrons a los 21 días de desarrollo (observado en lupa a 40X). E.- Huevo de Chicoreus brevifrons a los 21 días de desarrollo (observado en microscopio a 4X). F. y G.- Juveniles luego de la eclosión (15 días)

Fig. 3. A.- Chicoreus brevifrons ovigerous mass. B. and C.- Ovigerous mass capsules. D.- Chicoreus brevifrons egg at 21 days of development (observed at 40X magnifying glass). E.- Chicoreus brevifrons egg at 21 days of development (observed under microscope at 4X). F. and G.- Juveniles after hatching (15 days)

completamente desarrollada aun sin espinas bien formadas (Fig. 3-F y G).

La temperatura promedio de la laguna donde fueron extraídas las posturas es de $27^{\circ} \mathrm{C}$; esta se mantuvo en el acuario donde se desarrollaron los organismos dentro de las cápsulas, dado que Mostafa et al. (2013) sugieren que es importante controlar las condiciones ambientales en estudios de desarrollo embrionario, ya que juegan un papel relevante en el éxito de la eclosión $y$ en la tasa de crecimiento de los gasterópodos a cultivar. La cantidad de días necesarios para la eclosión de los individuos pertenecientes a la especie C. brevifrons fue de 45 días, siendo inferior a la reportada por Cabrera et al. (1990), quienes señalaron la eclosión para el día 52 desde la puesta de la masa ovígera y una talla inicial promedio de $2.22 \mathrm{~mm}$. Sin embargo, Mostafa et al. (2013) publicaron un total de 40 días para que suceda la eclosión de los organismos en estadio juvenil para la especie C. ramosus, lo que estaría indicando que este género presenta una posible variación en la tasa y periodo de la eclosión. La temperatura tiene influencia sobre el desarrollo larvario de los gasterópodos, interviniendo sobre la eclosión de los organismos (Góngora et al. 2007), por lo que el haber 
mantenido una temperatura constante de $27^{\circ} \mathrm{C}$ favoreció el desarrollo intracapsular y la supervivencia de los animales. A su vez, Cabrera et al. (1990) señalan que temperaturas inferiores al medio donde se desarrolla C. brevifrons retrasa el crecimiento del organismo dentro de la cápsula, haciéndolo más lento y prolongando el periodo de encapsulación, es por ello que probablemente se presentan diferencias entre los días de la eclosión del estudio antes nombrado (donde la temperatura fue de $22^{\circ} \mathrm{C}$ ) y la presente investigación. Sin embargo, hay que señalar que no se conoce el tiempo de duración del desarrollo intracapsular de la especie en su ambiente natural.

El impacto de la temperatura de cultivo en el desarrollo larvario de los gasterópodos ha sido registrado en otras especies de Murícidos como: C. concholepas, Xanthochorus cassidiformis, Plicopurpura pansa y Murex pomum (Naegel \& Gómez del Prado, 2004); en todos los casos se concluye que una temperatura alta del agua acorta el periodo de desarrollo larvario, mientras que temperaturas bajas retrasan el tiempo de eclosión.

Otros factores, que influyen en la tasa de la eclosión y la velocidad de crecimiento son la disponibilidad de alimento, así como la presencia de huevos nutricios: en este estudio, la eclosión de los organismos se efectuó a un máximo de 45 días con individuos de tallas mínimas de 1.60 $\mathrm{mm}$. La velocidad de crecimiento de los organismos una vez fuera de las cápsulas se refleja principalmente en la longitud más que en el peso, debido al gasto de energía en la alimentación y en los periodos de crecimiento de la concha. Según Góngora et al. 2011, las diferencias en los tiempos de la eclosión pueden explicarse fundamentalmente por la temperatura de incubación y la alimentación, al igual que el tamaño y origen de los reproductores.

\section{CONCLUSIONES}

La proporción sexual general fue de 1:1, el número de hembras representaron el $58.79 \%$ y los machos el $41.21 \%$ del total de los individuos (381).

Las posturas estaban conformadas por un máximo de 165 cápsulas de color blanco recién puestas. A los 21 días de desarrollo se puede notar la presencia de loshuevosnutricios que sirven dealimento para los organismos desarrollados.

La eclosión se efectúa pasados los 45 días desde la puesta, eclosionando de 3 a 5 individuos por cápsula.

\section{BIBLIOGRAFÍA}

Baqueiro, E. \& Aldana, D. (2003). Patrones en la biología poblacional de moluscos de importancia comercial en México. Rev. Biol. Trop., 51(4), 97-107.

Cabrera, T., Troccoli, L. \& León, L. (1990). Algunos aspectos sobre la biología del chivato (Chicoreus brevifrons L.) (Mollusca: Gastropoda: Muricidae). Saber, 3(2), 31-34.

Cognetti, G., Sará, M. \& Magazzú, G. (2008). Biología marina. Milano, Italia: Calderini.

D’Armas, H., Yánez, D., Reyes, D. \& Salazar, D. (2009). Composición de ácidos grasos de los caracoles marinos Phyllonotus pomum y Chicoreus 
brevifrons (Gastropoda: Muricidae). Rev. Biol. Trop., 58(2), 645-654. doi: 10.15517/rbt.v58i2.5235.

Díaz, J. \& Puyana, M. (1994). Moluscos del Caribe colombiano (1era. ed.). Santa Fe, Bogotá: Editorial Presencia. Galindo, L. (2009). Estudio morfométrico del dimorfismo sexual de las conchas de especies de gastrópodos marinos comunes (Mollusca: Gastropoda) en Venezuela. Tesis de doctorado no publicada, Universidad de Oriente, $\mathrm{Cu}$ maná, Venezuela.

Góngora, A., Domínguez, A., Muñoz, A. \& Rodríguez, L. (2007). Obtención de masas ovígeras del caracol lancetilla, Strombus pugilis (Mesogastropoda: Strombidae) en condiciones de laboratorio. Rev. Biol. Trop., 55(1), 183-188.

Góngora, A., García, M., Domínguez, A. y Camacho, F. (2011). Aspectos reproductivos cuantitativos del caracol murex negro, Hexaplex nigritus (Phillippi, 1845) en condiciones de laboratorio. Ciencia y Mar, XV(44), 31-34. González, A. (1970). Posibilidad del cultivo de moluscos, incluyendo el estudio de las diferentes etapas de la ostricultura en la Isla de Margarita. Venezuela: CIC. UDO-NE.

Itriago, M. (1977). Estudio citogenético y anatómico de las especies Chicoreus brevifrons (Lamarck, 1822) y Chicoreus pomum (Gmelin, 1791) (Neogastropoda Muricidae). Tesis de pregrado no publicada, Universidad de Oriente, Cumaná, Venezuela.

Leiva, G., Muñoz, J. \& Navarro, J. (1998). Desarrollo intracapsular y mecanismos de eclosión del caracol trumulco Chorus giganteus (Gastropoda: Muricidae), bajo condiciones de laboratorio. Rev. Chil. Hist. Nat., 71, 157-167.
Marval, H. (1981). Contribución a la biología de Chicoreus brevifrons (Lamarck, 1822) y Chicoreus pomun (Gmelin, 1781): Estudio comparativo del tracto digestivo. Tesis de pregrado no publicada, Universidad de Oriente, Cumaná, Venezuela.

Miloslavich, P., Penchaszadeh, P. \& Bigatti, G. (2007). Imposex en gasterópodos de Venezuela. Cienc. Mar, 33(003), 319-324.

Morfín, J. (2000). Ecología y aprovechamiento del caracol del tinte Plicopurpura pansa en las costas del Pacífico Mexicano. Tesis de doctorado no publicada, Politécnico Nacional, La Paz, México.

Mostafa, M., Tarek, M. \& Mohamed, Y. (2013). Spawning frequency, larval development and growth of Muricid gastropod Chicoreus ramosus (Linnaeus, 1758) in the laboratory at Hurghada, Northern Red Sea, Egypt. Egypt. J. Aquatic. Res., 39, 125-131. doi: 10.1016/j.ejar.2013.07.002.

Naegel, L. \& Gómez del Prado, P. (2004). Embriogénesis y desarrollo larvario intracapsular de Plicopurpura pansa (Gould, 1953) (Prosobranchia, Muricidae) en condiciones de laboratorio. Cienc. Mar., 30(2), 297-310.

Nieto-Bernal, R., Rodríguez, A., Chasqui, L., Castro, E. \& Gil-Agudelo, D. L. (2011). Distribución y abundancia de las poblaciones de gasterópodos de importancia comercial en La Guajira, Caribe colombiano. Bogotá, Colombia: INVEMAR Alianza Ediprint Ltda.-Guerra.

Olabarria, C. (1999). Estructura y variación estacional de poblaciones de moluscos asociadas a la pesca artesanal de langosta en el Pacífico Tropical. Rev. Biol. Trop., 47(4), 851-865.

Ordaz, G., D’Armas, H., Yáñez, D., Hernández, J. \& Camacho, A. (2010). 
Metabolitos secundarios, letalidad $\mathrm{y}$ actividad antimicrobiana de los extractos de tres corales y tres moluscos marinos de Sucre, Venezuela. Rev. Biol. Trop., 58(2), 677-688.

Prüsmann, J. \& Palacio, J. (2008). Colonización de moluscos y crustáceos en raíces de mangle rojo en una laguna costera de la punta norte del golfo de Morrosquillo. Gestión y Ambiente, 11(3), 77-86.

Quinceno, P. \& Palacio, J. (2008). Aporte al conocimiento de los macroinvertebrados asociados a las raíces del mangle (Rizophora mangle) en la ciénaga la
Boquilla, municipio de San Onofre, Sucre. Gestión y Ambiente, 11(3), 67-78.

Ramírez, P. (1996). Lagunas costeras venezolanas. Porlamar, Venezuela: Benavente \& Martínez CA.

Román, G., Martínez, G., García, O. \& Freites, L. (2001). Reproducción. En A. Maeda-Martínez (Ed.), Los moluscos pectínidos de Iberoamérica: ciencia y acuicultura (pp. 27-59). México D. F.: Limusa.

Tresierra, A. \& Culquichicón, Z. (1993). Biología pesquera. Trujillo, Perú: Libertad.

Zar, J. H. (1996). Biostatistical analysis. New Jersey, EE. UU.: Prentice-Hall Inc. 